\section{Living well with asthma}

Michael Freedman, Samuel Rosenberg and Cynthia Divino. New York, The Guildford Press 1998.

'There are no happy or sad events. How we look at and think about these events will determine whether they are happy or sad.'

This book could only be from the USA. Three clinical psychologists have got together to share their experiences of helping people deal with the predicament of having asthma. They worked at the National Jewish Hospital, a major asthma centre in Denver, Colorado, and many of the people whose stories illustrate the book are at the more severe end of the asthma spectrum.

There is a great deal of practical wisdom on how to help people handle those aspects of chronic illness to which many doctors and nurses give inadequate attention: the emotional response to ill health; the difficulties posed for relationships with partner and family; the impact on sex life; the threat to employment. Simple cognitive, behavioural and family therapy techniques to address these problems are presented as strategies for change. There is also a chapter on problems with brusque unsympathetic physicians, and their fees.

I would happily recommend this book to anyone having substantial trouble from their asthma - with a warning about the American flavor. I came away from the book with some good ideas on how to help my patients live with their asthma, and with renewed gratitude to be practising medicine in the British NHS rather than in the land of the free where health care doesn't come cheap.

Duncan Keeley

General Practitioner, Oxfordshire

Abstract: Erratum

Due to an oversight on the part of the authors an incorrect abstract (Charlton I. A randomised control study to assess the effectiveness of a GP and Asthma Nurse education session with Australian primary school teachers. Asthma in Gen Pract 1998; 6(Suppl 1): S2) was published in the Annual Scientific Meeting Supplement. A corrected abstract is published here.

\title{
A controlled study to assess the effectiveness of a general practitioner and asthma nurse education session with Australian primary school teachers.
}

I Charlton and *M Mullee, Kincumber, Australia and *University of Southampton, Hampshire.

\section{INTRODUCTION}

The management of asthma in Australian schools remains a problem despite the recent publication of guidelines by the Australian Thoracic Society.

\section{AIMS OF THE STUDY}

The project wished to evaluate the effectiveness of a one hour education programme conducted by a local GP and Asthma Nurse with a group of primary school teachers.

\section{METHODS}

Following a meeting with the headmasters of the New South Wales Department of Education, Central Coast Region, five schools were randomly selected to undergo an intervention programme conducted by the local GP and Asthma Nurse. Intervention schools were matched with schools of a similar size and location and whether they had received asthma education in the past. Questionnaires evaluating knowledge, attitudes and response to scenarios were administered before and after the intervention to both the control and intervention schools.

\section{INTERVENTION}

A one hour tutorial outlining the basic mechanisms of asthma, use of inhalers and spacers and treatment of emergency episodes was undertaken. Ample time was provided for questions and answers.
Intervention schools were provided with an asthma first aid kit, wall posters and reading material. The control schools received no contact other than to complete the before and after questionnaires.

\section{RESULTS}

Seventy two teachers participated in the intervention and 60 teachers provided information for the control group. Knowledge scores prior to the intervention were high, with only a small and non-significant gain as a result of the intervention. A significant difference between the intervention and control group was recorded for the teacher's awareness of the school's asthma policy $(\mathrm{p}<0.03)$.

A higher proportion of teachers in the intervention group were able to provide a suitable response to the scenario questions after the intervention compared to the control group $(\mathrm{p}<0.07)$. However, after the intervention, $68 \%$ of the teachers were still unable to identify the appropriate steps to take when salbutamol therapy failed to be effective.

\section{ConClusions}

This controlled study outlines the difficulty of developing programmes to improve teachers management of asthma in the school situation. Programmes that involve a more active participation in the learning process may be more effective rather than the seminar approach used in this study. 